Copyright (C) 2013 IEEE. Personal use of this material is permitted. Permission from IEEE must be obtained for all other uses, in any current or future media, including reprinting/republishing this material for advertising or promotional purposes, creating new collective works, for resale or redistribution to servers or lists, or reuse of any copyrighted component of this work in other works. 


\title{
Operation and Control of Three Phase Microgrids Consisting of Single-Phase DERs
}

\author{
Ruwan P.S. Chandrasena, Farhad Shahnia, Sumedha Rajakaruna and Arindam Ghosh
}

\begin{abstract}
Autonomous operation of a three-phase microgrid (MG) consisting of arbitrarily located single-phase distributed energy resources (DER) among the three phases is investigated. Due to random connection of single-phase loads and DERs to different phases, it is highly probable that one phase has more generation while another phase has more demand. This becomes a challenging issue during autonomous operation. This paper demonstrates how single-phase DERs in one phase can share the load demand in other phases. Several case studies are carried out using PSCAD/EMTDC to demonstrate the efficacy of the proposed power management and control strategy for single-phase DER converters distributed unequally among the three-phases in the MG.
\end{abstract}

Index Terms - Microgrid, single-phase DER, Power Sharing.

\section{INTRODUCTION}

The ever increasing energy demand, environmental concerns, the necessity of cost reduction and higher reliability requirements are the driving force behind the motivation of the modern power systems towards the distributed generation [12]. In near future, it is expected that majority of houses will have a single-phase DER connected to their network. Hence, a group of interconnected neighbor houses form a network which can operate in autonomous mode in the event of a grid failure, referred as a MG. The MG should appear as a single controllable unit that can respond to system changes quickly [3]. In a MG, parallel DERs are controlled to deliver the desired active and reactive power to the system while local signals are used as feedback to control the converters. The power sharing among the DERs can be achieved by controlling two independent quantities, magnitude and the frequency of fundamental voltage, at the converter output [4-7].

The MG concept has become well-established and ample of literature could be found on power management and control in three-phase MG systems. Different power management strategies and controlling algorithms for a MG is proposed in [8-10]. The control and power sharing of parallel-connected threephase DERs are extensively studied [11-13]. In the presence of unbalanced and nonlinear loads, the converters can operate in current-controlled [14] or voltage-controlled mode [15]. In current-controlled mode, the output current reference for each converter should be properly calculated based on the load demand and the desired power sharing ratio among the existing DERs. This can be relatively complex process when the MGs are supplying single-phase, unbalanced or harmonic loads as they should share the active and reactive power as well as harmonic, negative and zero sequence currents. This needs a fast

R.P.S. Chandrasena and S. Rajakaruna are with the Department of Electrical and Computer Engineering, Curtin University, Perth, Australia

(ruwan.chandrasena@curtin.edu.au,s.rajakaruna@curtin.edu.au).

F. Shahnia is with the Centre of Smart Grid and Sustainable Power Systems, Department of Electrical and Computer Engineering, Curtin University, Perth, Australia (farhad.shahnia@curtin.edu.au).

A. Ghosh is with the School of Electrical Engineering and Computer Science, Queensland University of Technology, Australia (a.ghosh@qut.edu.au). and accurate sequence and harmonic current extraction mechanism as discussed in [14]. However, similar results can be achieved using a voltage control mode without any sequence and harmonic current extractions as discussed in [16].

However, the operation of the single-phase DERs in a three-phase MG, especially in autonomous mode, is yet to be explored. This paper focuses on operation and control of single-phase DERs in a three-phase MG where a single-phase DER in one phase is responding to load changes in all three phases of the MG. In this paper, it is assumed the single-phase DERs are arbitrarily connected to different phases of the threephase MG. Due to the random connection of single-phase DERs and single-phase loads, there is a high probability to have one phase with extra generation capacity while other phases with extra demand. This is of high importance in MG autonomous operation. Hence, in autonomous mode, the surplus power generation of the single-phase DERs should be circulated through the distribution transformer to other phases. Simulation studies are carried out in PSCAD/EMTDC to validate the performance of the proposed power management.

The rest of this paper is arranged as follows. Section II describes the structure and modeling of the MG under consideration. Section III discusses the developed MG control strategies. Simulation results are demonstrated and discussed in Section IV to demonstrate the performance of the developed control and power management strategy.

\section{MICROGRID STRUCTURE AND MODELING}

Let us consider the MG formed by a group of neighbour residential houses with some of them having single-phase converter-interfaced DERs, as shown Fig. 1. Let us assume that in this MG four houses have single-phase DERs. The MG is assumed to be supplied by a Dyn type, three-phase, $11 \mathrm{kV} / 400 \mathrm{~V}$ distribution transformer, which is a common type of distribution transformers in Australia. The considered DERs are operating in voltage-controlled mode. A Distribution Static Compensator (DSTATCOM) is installed at the secondary side of the distribution transformer in the MG to regulate the voltage at their Point of Common Coupling (PCC). The DER and DSTATCOM converter structure are discussed in detail below.

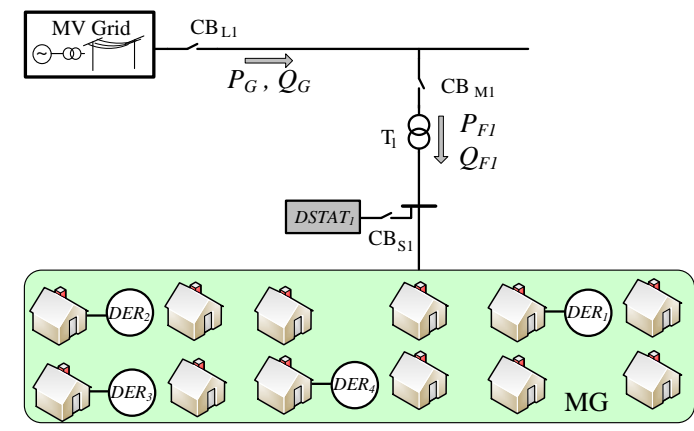

Fig. 1. Schematic diagram of the network under consideration. 


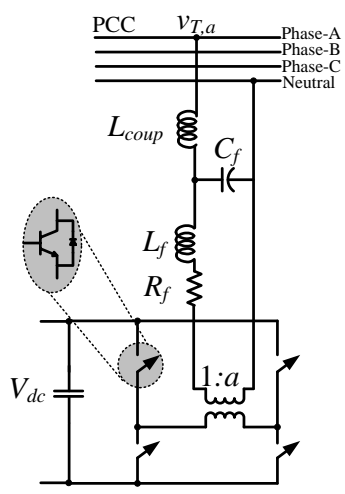

(a)

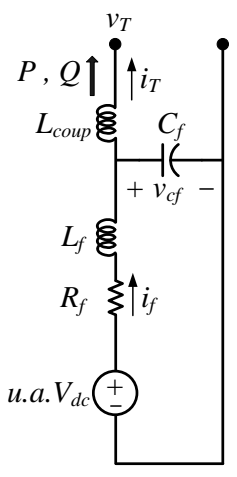

(b)
Fig. 2. (a) Schematic diagram of the DER converter and filter,

(b) Single-phase equivalent circuit of the converter and filter.

The DERs are connected to the MG through Voltage Source Converters (VSC). The converters consist of a single-phase $\mathrm{H}-$ bridge, using IGBTs, as shown in Fig. 2(a). Each IGBT is equipped with proper parallel reverse diode, snubber and protection circuits. The output of $\mathrm{H}$-bridge is connected to a single-phase transformer, with 1: $a$ turns ratio. The transformer can provide galvanic isolation and voltage boosting. In this figure, the resistance $R_{f}$ represents the switching and transformer losses, while the inductance $L_{f}$ represents the leakage reactance of the transformer and the filter capacitor $C_{f}$ is connected to the output of the transformer to bypass the switching harmonics. The single-phase equivalent circuit of the DER converter and filter is shown in Fig. 2(b).

The three-phase DSTATCOM connected to control the voltage at the PCC consists of three single-phase $\mathrm{H}$-bridges similar to the one of DERs. The outputs of three single-phase transformers are star-connected.

\section{MICROGRID CONTROL STRATEGIES}

MG control consists of several modules, namely synchronization/islanding detection, DER converter output power control, converter switching control and load shedding modules. These modules are described in detail, below.

\subsection{Synchronization/Islanding Module}

Depending on the state of $\mathrm{CB}_{\mathrm{M} 1}$, the MG operates in either grid-connected or off-grid mode. In this study, the DER converters are controlled on constant PQ mode when the MG is in grid-connected mode and in droop control during off-grid mode. Hence, islanding detection technique is required to identify the MG mode of operation and to select the proper control algorithm accordingly. In this study, islanding detection is based on monitoring $\mathrm{CB}_{\mathrm{M} 1}$ status and the assumption of communication interface availability to transfer this information to the DERs.

After maintenance/fault clearance in the MV network, the MG can reconnect to the grid. Proper resynchronization of the MG should be carried out before reconnection. In this paper, resynchronization is carried out by monitoring the magnitude and angle of the voltages in both sides of $\mathrm{CB}_{\mathrm{M} 1}$ through a Phased-Locked Loop (PLL). $\mathrm{CB}_{\mathrm{M} 1}$ closes when the difference between voltage magnitudes and angles in its two sides becomes less than a small pre-defined value. A similar synchronization is utilized for interconnecting a voltage-controlled DER to the MG.

\subsection{Power Output Control Module}

DER converters operate in constant PQ mode in gridconnected mode and in droop control in off-grid mode. For this, two different converter output voltage reference generations are required.

In grid-connected mode, the grid dictates the network voltage and frequency. Hence, the DERs are expected to generate the power at their rated capacities. In such a case, from Fig. 2(b), the instantaneous active $(p)$ and reactive power $(q)$ output of DERs can be expressed as

$$
\begin{aligned}
& p=\frac{\left|V_{T}\right| \times\left|V_{c f}\right|}{\omega L_{T}} \sin \left(\delta_{c f}-\delta_{T}\right) \\
& q=\frac{\left|V_{T}\right|}{\omega L_{T}}\left(\left|V_{c f}\right| \cos \left(\delta_{c f}-\delta_{T}\right)-\left|V_{T}\right|\right)
\end{aligned}
$$

where $V_{T}$ is the voltage at converter $\mathrm{PCC}, V_{c f}$ is the voltage across the AC filter capacitor and $V=|V| \angle \delta$ is the phasor representation of $v(t)$. The average active power $(P)$ and reactive power $(Q)$ can be derived by passing their instantaneous values through a low pass filter. Assuming the rated values for the active and reactive power and by monitoring the PCC voltage, the desired $v_{c f}$ can be calculated using (1). This will be the voltage reference for the converter output in grid-connected mode in Fig. 3.

In off-grid mode, the DERs vary their output voltage and frequency based on the droop control and pre-defined droop coefficients $(m \& n)$ to regulate the network voltage and frequency within acceptable limits, while sharing the loads in the network. Hence, the active and reactive power delivered by the DERs are measured and used to derive the reference DER converter output voltage as [17]

$$
\begin{aligned}
& \delta_{c f}=\delta_{\text {rated }}-m\left[\frac{X_{\text {line }}}{Z_{\text {line }}}\left(P_{\text {rated }}-P\right)-\frac{R_{\text {line }}}{Z_{\text {line }}}\left(Q_{\text {rated }}-Q\right)\right] \\
& \left|V_{\text {cf }}\right|=V_{\text {rated }}-n\left[\frac{R_{\text {line }}}{Z_{\text {line }}}\left(P_{\text {rated }}-P\right)+\frac{X_{\text {line }}}{Z_{\text {line }}}\left(Q_{\text {rated }}-Q\right)\right]
\end{aligned}
$$

where the LV feeder impedance is $Z_{\text {line }}=R_{\text {line }}+j X_{\text {line }}$ and the suffix rated shows the rated values. This will be the voltage reference for the converter in off-grid mode in Fig. 3.

Note that the synchronization module issues a command to the reference selection block to select the proper reference.

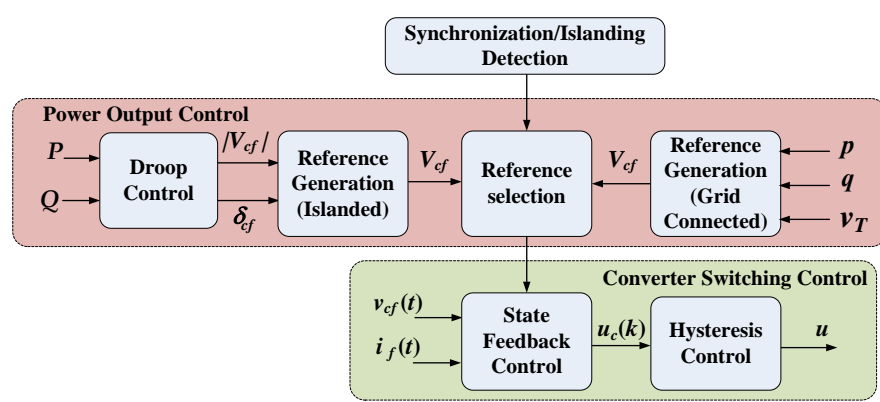

Fig. 3. Schematic block diagram of the MG control modules.

\subsection{Converter Switching Control Module}

Converter switching control module is responsible for proper turn on/off of the IGBTs such that the desired reference voltage is generated across the AC filter capacitor in both operation modes. In this study, a closed-loop optimal linear robust controller based on state feedback control is used to generate the 
switching function $u$. Assuming a bi-polar switching for the IGBTs, $u$ can take \pm 1 value which will subsequently turn on/off proper pair of IGBTs in the converter.

The bilinear differential equations that describe dynamic behavior of converter and filter can be given as

$$
\begin{aligned}
& \text { u.a.Vdc }=R_{f} i_{f}+L_{f} \frac{d i_{f}}{d t}+v_{c f} \\
& i_{f}=C_{f} \frac{d v_{c f}}{d t}+i_{T}
\end{aligned}
$$

Let us assume the state vector $x(t)$ for a DER be defined as

$$
x(t)=\left[\begin{array}{ll}
v_{c f}(t) & i_{f}(t)
\end{array}\right]^{T}
$$

where $i_{f}(t)$ is the current of filter inductor $L_{f}$ and $T$ is the transpose operator. Then, VSC equivalent circuit can be represented with state space description as

$$
\dot{x}(t)=A x(t)+B_{1} u_{c}(t)+B_{2} i_{T}(t)
$$

where

$$
A=\left[\begin{array}{cc}
0 & \frac{1}{C_{f}} \\
-\frac{1}{L_{f}} & -\frac{R_{f}}{L_{f}}
\end{array}\right] \quad B_{1}=\left[\begin{array}{c}
0 \\
\frac{a \cdot V d c}{L_{f}}
\end{array}\right] \quad B_{2}=\left[\begin{array}{c}
-\frac{1}{C_{f}} \\
0
\end{array}\right]
$$

while $u_{c}(t)$ is the continuous-time version of switching function $u$. Note that $i_{T}$ represents the load change effect on the DER control and hence is assumed as a disturbance to the control system. Eq. (5) can be represented in discrete-time domain as

$$
x(k+1)=F x(k)+G_{1} u_{c}(k)+G_{2} i_{T}(k)
$$

where

$$
F=e^{A^{\prime} \cdot T_{s}} \quad, \quad G_{1}=\int_{0}^{T_{s}} e^{A^{\prime} t} B_{1}^{\prime} d t \quad, \quad G_{2}=\int_{0}^{T_{s}} e^{A^{\prime} t} B_{2}^{\prime} d t
$$

and $T_{s}$ is the sampling time. From $(8), u_{c}(k)$ can be computed using a suitable state feedback control law as

$$
u_{c}(k)=-K\left[x^{\prime}(k)-x_{r e f}^{\prime}(k)\right]
$$

where $K=\left[k_{1} k_{2}\right]$ is the gain matrix and $x_{r e f}^{\prime}$ is the desired state vector for (4) in discrete-time domain.

As the system behavior in steady-state is interested and assuming a full control over $u_{c}(k)$, an infinite time Linear Quadratic Regulator (LQR) [18] is designed for this problem to define $K$. The LQR method ensures the desired results for the system while the variations of system load and source parameters are within acceptable limits.

Eq. (9) shows the total reference tracking error of each DER. The tracking error can be minimized by limiting that within a very small bandwidth (e.g. $\mathrm{h}=10^{-4}$ ). From (9) the switching function $u$, for each DER, is generated using a hysteresis control based on the error level as

$$
\begin{aligned}
& \text { If } \quad u_{c}(k)>+h \text { then } u=+1 \\
& \text { If }-h \leq u_{c}(k) \leq+h \text { then } u=\text { previous } u \\
& \text { If } \quad u_{c}(k)<-h \text { then } u=-1
\end{aligned}
$$

\subsection{Voltage Control}

According to the selected operational strategy, none of the DERs in the MG is responsible for MG voltage regulation in autonomous mode. Hence, a dedicated DSTATCOM with the structure describe in Section II is used to regulate the MG voltage. Since, the DSTATCOM structure is the same as that of the single-phase DER; a similar control system is deployed. More detail on DSTATCOM control is provided in [10].

\subsection{Load Shedding Module}

A three level load-shedding algorithm is developed in this study which monitors the frequency in every phase separately and if the frequency drops below the limit in one phase, it will apply the first level of load-shedding in that phase after a short delay time (i.e. 0.2 second in this study). This may bring the frequency of the respective phase into the acceptable range. Otherwise, the second load-shedding will be applied after a long delay time (e.g. 1 second in this study). In a similar way, the third load shedding will be applied after another long delay time, in case the second load-shedding fails to recover the phase-frequency.

\section{STUdy CASES AND Simulation Results}

In order to investigate the performance of the single-phase DERs in a three-phase MG, with the proposed control strategy, several case studies are carried out using PSCAD/EMTDC. It is to be noted that the MG is supplied through a three-phase 11 $\mathrm{kV} / 400 \mathrm{~V}$ distribution transformer. The parameters of network, DER converters and filters, DSTATCOM, loads and droop control coefficients are given in the Appendix.

\subsection{Grid-connected and Autonomous Operation}

First, let us investigate the dynamic performance of the DER converters of a MG for load changes. It is assumed in the system shown in Fig. $1, \mathrm{CB}_{\mathrm{G}}, \mathrm{CB}_{\mathrm{M} 1}$ and $\mathrm{CB}_{\mathrm{S} 1}$ are closed. The system is in steady state condition at $t=0 \mathrm{~s}$ and all the DERs are running in their rated condition.

At $t=0 \mathrm{~s}$, the MG is operating in grid-connected mode and the surplus of DERs generation is $2.4 \mathrm{~kW}$ and flowing into the grid. Let us assume at $t=1 \mathrm{~s}$, the MG disconnects from the grid. As Fig. 4(a) shows, the total network active power demand remains the same after grid is disconnected and this demand is supplied by the DERs of the MG. As Fig. 4(b) shows, at $t=1 \mathrm{~s}$, the DERs active power generation drops as the grid is disconnected, since no power is delivered to the grid at this time.

Figs. 4(c) and (d) show the active power flow in the individual phases at primary and secondary sides of the distribution transformer. It can be seen that, different phases carry different amounts of power due to the unbalanced distribution of DERs and loads in the MG. During $1<t<2$, MG has $5 \mathrm{~kW}$ of demand in each phase with $2.8 \mathrm{~kW}, 8.0 \mathrm{~kW}$ and $4.2 \mathrm{~kW}$ generations in phases $-\mathrm{A}, \mathrm{B}$ and $\mathrm{C}$, respectively. Hence, phase- $\mathrm{B}$ is having excess of generation whereas phase- $\mathrm{A}$ and $\mathrm{C}$ are having lack of generation. As seen from Fig. 4(c), after the grid is disconnected, the power in the three phases in transformer primary become zero; however, there is a $2,0.8$ and $-2.8 \mathrm{~kW}$ of power flows in phases $\mathrm{A}, \mathrm{C}$ and $\mathrm{B}$ at the transformer secondary, respectively. This shows a power circulation through the 
distribution transformer in its secondary from phase-B to the other phases. This way, the single-phase DERs in phase-B can contribute to the load demand in other phases, too.

At $t=2 \mathrm{~s}$, a balanced load increase of $60 \%$ (i.e. $9 \mathrm{~kW}$ ) is applied in the MG. As Fig. 4(b) shows, all the DERs in the MG share the load increase proportional to their ratings.

At $t=3 \mathrm{~s}$, a single-phase load increase of $13.3 \%$ (i.e. $2 \mathrm{~kW}$ ) in phase-A is applied. As Fig. 4(b) shows, all the DERs increases their output power to share this load increase and DER-3 connected to phase-B shares a large portion of the load due to its higher capacity.

At $t=4 \mathrm{~s}, 4 \mathrm{~kW}$ of single phase load is connected to phase$\mathrm{B}$ and at $t=5 \mathrm{~s}, 1 \mathrm{~kW}$ of load connected to phase- $\mathrm{C}$ is dropped. As figure shows, each of these load changes are shared by the DERs connected to different phases. Since the transformer primary side is open during $1<t<6 \mathrm{~s}$, its power in the primary side remains zero, as shown in Fig. 4(c). However, as it can be seen from Fig. 4(d), phase-B is responding to the load changes in the other phases through power circulation take place across the distribution transformer.

Fig. 4(e) shows the MG frequency during those load transitions and as figure shows, the MG frequency remains within the acceptable limits.

(a)

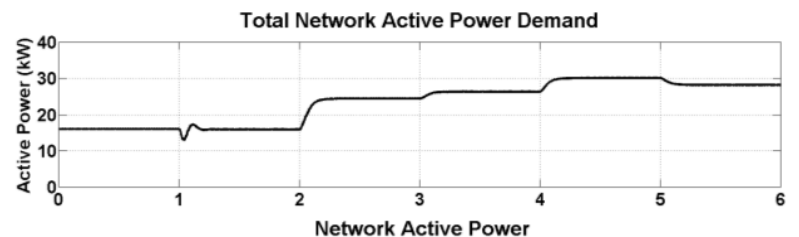

(b)

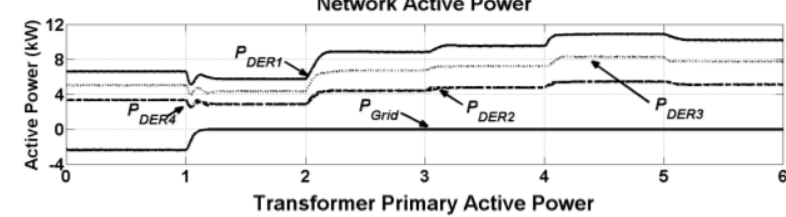

(c)

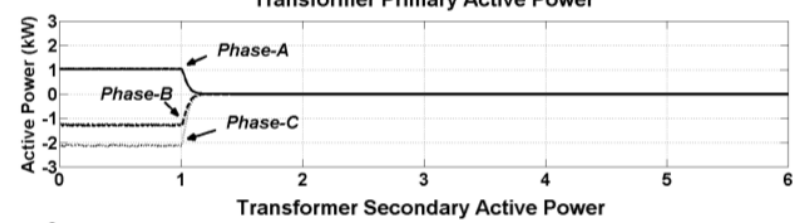

(d)

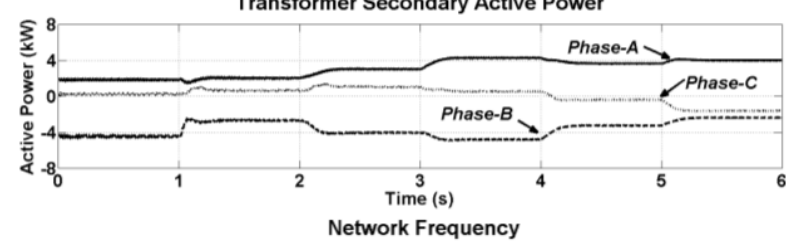

(e)

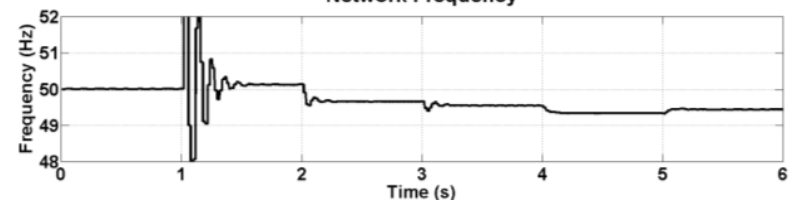

Fig. 4. Simulation results of MG for case 4.1:
(a) Total network active power demand,
(b) Active power flow of the DERs and the grid,
(c) Transformer primary active power,
(d) Transformer secondary active power,
(e) The network frequency.

\subsection{DER and MG Isolation and Resynchronization}

Let us now investigate the dynamic performance of DER converters during transition from grid-connected to autonomous as well as autonomous to grid-connected mode. The DER isolation and resynchronization to the MG is also investigated in this section.
Let us assume the MG is grid-connected and operates in steady state condition at $t=0 \mathrm{~s}$ which is disconnected from the grid at $t=1 \mathrm{~s}$. Later, at $t=2 \mathrm{~s} \mathrm{DER}-3$ which is connected to phase-C and supplies $28 \%$ of load demand (i.e. $4.3 \mathrm{~kW}$ ) is isolated from the MG. At $t=3 \mathrm{~s}$, the resynchronization of DER-3 to the MG is initiated and at $t=3.68 \mathrm{~s}$, it is connected back to the MG. At $t=4 \mathrm{~s}$, resynchronization of the MG to the main grid is activated and at $t=4.15 \mathrm{~s}$, it is resynchronized and reconnected to the grid.

As shown in Fig. 5(a), since DER-3 is disconnected at $t=2$ $\mathrm{s}$, the power supplied by DER-3 drops to zero and DER 1-2 and DER-4 increase their generation proportional to the desired power sharing ratio to match the local demand.

At $t=3 \mathrm{~s}$, the resynchronization of DER-3 to the MG is initiated. Fig. 5(b) shows phase-A voltage on either side of the DER-3 circuit breaker. As Fig. 5(b) shows, DER-3 synchronizes with MG at $t=3.68 \mathrm{~s}$ and the voltages of the two sides of the circuit breaker become in phase.

Similarly, at $t=4 \mathrm{~s}$, the resynchronization of MG with the grid is initiated and at $t=4.15 \mathrm{~s}, \mathrm{MG}$ is reconnected to the grid. Fig. 5(c) shows phase-A voltage on either side of the $C_{M 1}$ which confirms proper resynchronization at $t=4.15 \mathrm{~s}$. After MG is reconnected to the grid, DERs operate at their rated capacity which makes the grid to absorb $2.2 \mathrm{~kW}$.

(a)

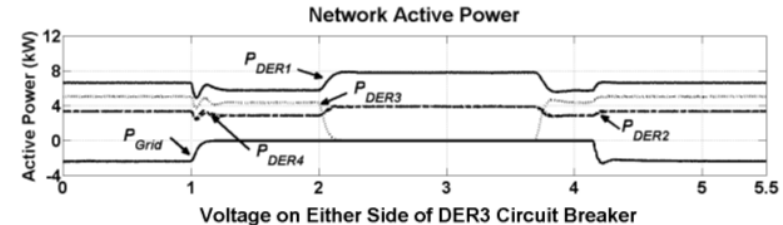

Voltage on Either Side of DER3 Circuit Breaker

(b)

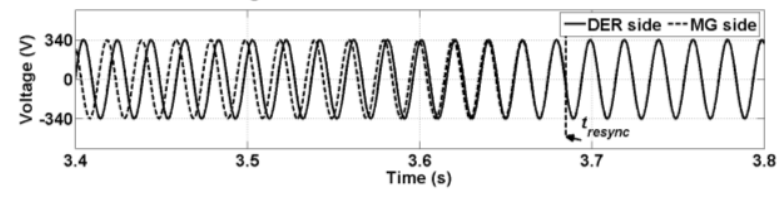

Voltage on Either Side of MG main Circuit Breaker

(c)

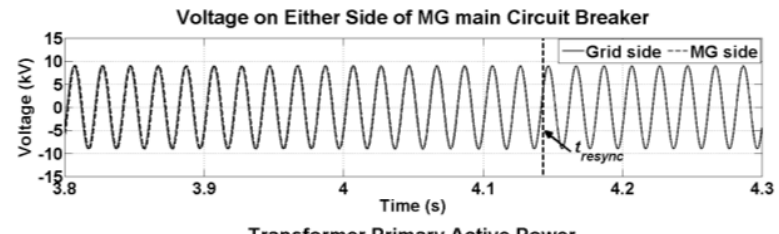

(d)

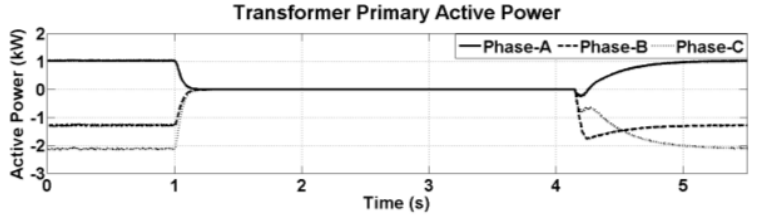

Transformer Secondary Active Power

(e)

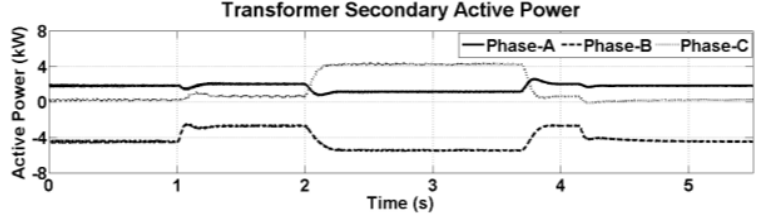

Fig. 5. Simulation results of MG for case 4.2:
(a) Active power flow in the MG,
(b) Voltage on either side of DER3 circuit breaker,
(c) Voltage on either side of the $\mathrm{CB}_{\mathrm{M} 1}$,
(d) Transformer primary active power,
(e) Transformer secondary active power.

Power flows across the distribution transformer primary and secondary are shown in Figs. 5(d) and (e), respectively. As shown in Fig. 5(d), power flows across the transformer primary become zero during $1<t<4.2 \mathrm{~s}$, where it is opened. However, during the same period, power circulation takes place in the 
secondary of the transformer as shown in the figure. As DER-3 which is the only generator connected to phase-C, disconnects at $t=2 \mathrm{~s}$, the DERs connected to other phases supply the loads on phase-C. As, it can be seen in the figure, DERs connected to phase-B supply about $20 \%$ (i.e. $1 \mathrm{~kW}$ ) of the total load demand in phase-A and $100 \%$ of the total load demand on phase- $\mathrm{C}$.

\subsection{Load shedding}

The aforementioned power management becomes possible due to the delta/star-grounded connection of the distribution transformer. This connection type supports the zero sequence current circulation. Now, let us consider case 4.1, in which the distribution transformer is isolated from both sides when the MG falls into off-grid mode.

When the distribution transformer is disconnected from both sides, there is no path for zero sequence current to circulate and power circulation is no longer available. Therefore, in such a case, the three phases of the MG will operate as three individual networks while the DERs in each phase only supply the load demand in that phase, as shown in Fig. 6.

Let us assume, Phase-A has $5 \mathrm{~kW}$ of demand whereas the rated capacity of DER-4 which is connected to this phase is only $3.3 \mathrm{~kW}$. Hence, as shown in Fig. 6(b), the frequency in phase-A drops to $49.4 \mathrm{~Hz}$. Similarly, phase-B has $9.9 \mathrm{~kW}$ of rated generation capacity by DER-1 and DER-2 while its load demand is $5 \mathrm{~kW}$. Hence, the frequency in phase-B increases to $50.5 \mathrm{~Hz}$.

At $t=2 \mathrm{~s}$, a load increase of $60 \%$ is applied for each phase and, as it is shown in Figs. 6(a) and (b), generation in each phase increases and the operating frequency drops. At $t=3 \mathrm{~s}$, a $2 \mathrm{~kW}$ load is increased in phase- $\mathrm{A}$ is applied and hence the frequency drops to $48 \mathrm{~Hz}$, which is beyond the acceptable limit for frequency variation.

(a)

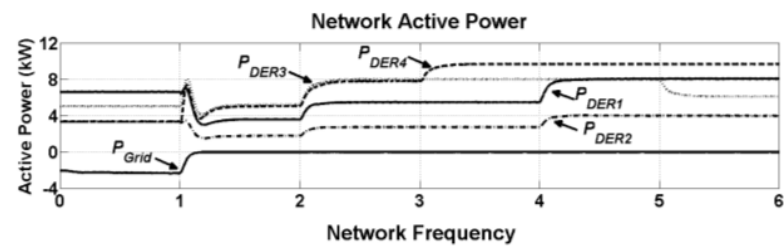

(b)
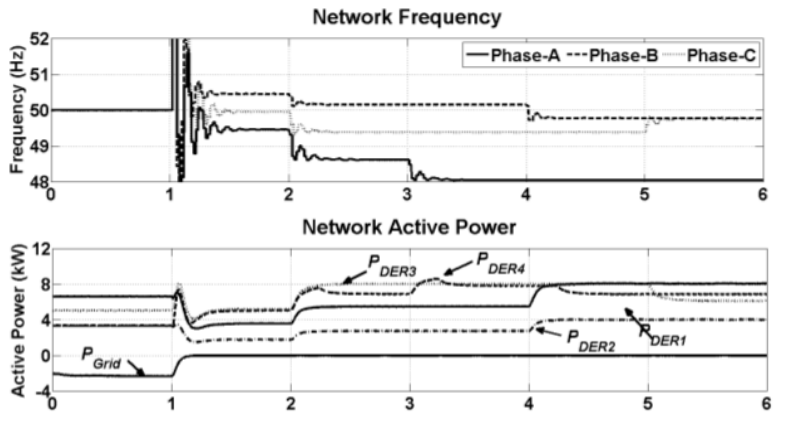

(c)

Network Frequency

(d)

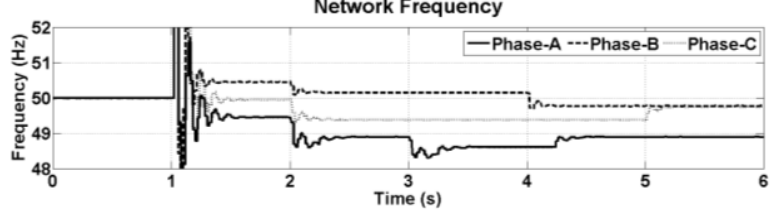

Fig. 6. Simulation results of MG for case 4.3:
(a) Network active power without load-shedding scheme,
(b) Network frequency without load-shedding scheme,
(c) Network active power with load-shedding scheme,
(d) Network frequency with load-shedding scheme.

Now, let us assume that the three level load-shedding algorithm, discussed in Section 3.4, is in operation. Fig. 6(c) shows the network active power flow and Fig. 6(d) shows the frequency variation of the three phases. At $t=2 \mathrm{~s}$ Phase-A experiences a sever (more than 2\%) frequency drop. Therefore, load-shedding scheme will shed the first set of low prioritized loads in Phase-A (equal to $1 \mathrm{~kW}$ ). However, shedding $1 \mathrm{~kW}$ of load is not effective and does not bring the frequency in PhaseA back to normal range. Hence, after another $1 \mathrm{~s}$, second level of load-shedding is applied. As the frequency is still below the minimum acceptable limit, at $t=3.2 \mathrm{~s}$, the third load-shedding is also applied. In Phase-B and Phase-C, no load shedding takes place since the frequencies in those phases remain within the assumed acceptable range. However, if the power circulation through the distribution transformer is available, the above load shedding was not required, as shown in Fig. 4(e).

\section{CONCLUSION}

Operation and control of converter-interfaced single-phase DERs, distributed unequally in a three-phase MG, in both grid- connected and autonomous modes were investigated. In such cases, it is highly probable that some phases have excess generation capability in their DERs and other phases have higher demand. In this paper, it was demonstrated that excess power generated by single-phase DERs in one phase is circulated from that phase to the other phases through the delta/stargrounded (or Dyn type) distribution transformer. Simulation results confirm that the proposed single-phase DER converter control strategy based on the power circulation concept through a Dyn transformer results in successful network performance in grid-connected as well as autonomous mode. It was also demonstrated that it reduces the necessity of loadshedding in the phases with less generation capacity in their DERs.

\section{APPENDIX}

Table I. Technical data of the network parameters of Fig. 1.

\begin{tabular}{ll} 
MV Network & $11 \mathrm{kV} \mathrm{L}-\mathrm{L}$ RMS, $50 \mathrm{~Hz}$ \\
MV Line Impedance & $R=0.2 \Omega, L=10 \mathrm{mH}$ \\
LV Feeder & $415 \mathrm{~V} \mathrm{~L}-\mathrm{L}$ RMS, $50 \mathrm{~Hz}$ \\
LV Line Impedance & $R=0.02 \Omega, L=1 \mathrm{mH}$ \\
Transformer & $30 \mathrm{kVA}, 11 \mathrm{kV} / 415 \mathrm{~V}$, Three-Phase, $50 \mathrm{~Hz}$, \\
& $\Delta / \mathrm{Y}$-Grounded, $Z_{I}=5 \%$ \\
Balanced Three Phase Loads & $P=15 \mathrm{~kW}, P F=0.95$ \\
Single Phase Load & $P=2 \mathrm{~kW}, P F=0.95$ in Phase-A \\
Single Phase Load & $P=4 \mathrm{~kW}, P F=0.95$ in Phase-B \\
Single Phase Load & $P=1 \mathrm{~kW}, \mathrm{PF}=0.95 \mathrm{in}$ PhaseC \\
DER VSCs and Filters & $R_{f}=0.1 \Omega, L f=0.36 \mathrm{mH}, C_{f}=50 \mu \mathrm{F}$, \\
& $V_{d c}=150 \mathrm{~V}, a=3.33, h=10^{-5}$ \\
DSTATCOM VSC and & $R_{f}=1 \mathrm{~m} \Omega, L_{f}=4 \mathrm{mH}, C_{f}=25 \mu \mathrm{F}, L_{T}=10$ \\
Filter & $\mathrm{mH}, V_{d c}=1 \mathrm{kV}, \mathrm{C}_{\mathrm{dc}}=2000 \mu \mathrm{F}, a=1, h=10^{-4}$ \\
\hline
\end{tabular}

Table II. Technical data of DERs and droop control coefficients in Fig. 1.

\begin{tabular}{lccccc}
\hline DER & $\begin{array}{c}\text { Rating } \\
{[\mathrm{kW}]}\end{array}$ & $\begin{array}{c}L_{T} \\
{[\mathrm{mH}]}\end{array}$ & $\begin{array}{c}m \\
{[\mathrm{rad} / \mathrm{kW}]}\end{array}$ & $\begin{array}{c}n \\
{[\mathrm{~V} / \mathrm{kVAr}]}\end{array}$ & $\begin{array}{c}\text { Phase } \\
\text { Connection }\end{array}$ \\
DER-1 & 6.6 & 3.4 & 0.95 & 0.54 & $\mathrm{~B}$ \\
DER-2 & 3.3 & 6.8 & 1.90 & 1.08 & $\mathrm{~B}$ \\
DER-3 & 5.0 & 4.5 & 1.26 & 0.72 & $\mathrm{C}$ \\
DER-4 & 3.3 & 6.8 & 1.90 & 1.08 & $\mathrm{~A}$ \\
\hline
\end{tabular}




\section{REFERENCES}

[1] B Kroposki, C. Pink, R. DeBlasio, H. Thomas, M. Simões and P.K. Sen, "Benefits of Power Electronic Interfaces for Distributed Energy Systems," IEEE Trans. on Energy Conversion, vol. 25, no. 3, pp. 901-908, 2010 .

[2] T. Senjyu, T. Nakaji, K. Uezato and T. Funabashi, "A hybrid power system using alternative energy facilities in isolated island," IEEE Trans. on Energy Conversion, vol. 20, no. 2, pp. 406-414, 2005.

[3] R.H. Lasseter, "MicroGrids," IEEE Power Engineering Society Winter Meeting, vol. 1, pp. 305- 308, 2002.

[4] A.G. Madureira and J.A. Pecas Lopes, "Coordinated voltage support in distribution networks with distributed generation and microgrids," IET Renewable Power Generation, vol. 3, no. 4, pp. 439-454, 2009.

[5] K. Pandiaraj, P. Taylor, N. Jenkins and C. Robb, "Distributed Load Control of Autonomous Renewable Energy Systems," IEEE Trans. on Energy Conversion, vol. 16, no. 1, pp. 14-19, 2001.

[6] R. Majumder, G. Ledwich, A. Ghosh, S. Chakrabarti and F. Zare, "Droop control of converter-interfaced microsources in rural distributed generation," IEEE Trans. on Power Delivery, vol. 25, no. 4, pp. 2768 2778, 2010.

[7] S.J. Ahn, J.W. Park, I.Y. Chung, S.I. Moon, S.H. Kang and S.R. Nam, "Power-sharing method of multiple distributed generators considering control modes and configurations of a microgrid," IEEE Trans. Power Delivery, vol. 25, no. 3, pp. 2007-2016, 2010.

[8] I.Y. Chung, W. Liu, D.A. Cartes, E.G. Collins and S.I. Moon, "Control methods of inverter-interfaced distributed generators in a microgrid system," IEEE Trans. on Industrial Application, vol. 46, no. 3, pp. 1078 1088, 2010.

[9] C.K. Sao and P.W. Lehn, "Control and power management of converter fed microgrids," IEEE Trans. on Power System. vol. 23, no. 3, pp. 1088-1098, 2008.
[10] F. Shahnia, R.P.S. Chandrasena, S. Rajakaruna and A. Ghosh, "Primary control level of parallel DER converters in system of multiple interconnected autonomous microgrids within self-healing networks," IET Generation Transmission and Distribution, Accepted for publication. 2013.

[11] S.J. Ahn, J.W. Park, I.Y. Chung, S.I. Moon, S.H. Kang and S.R. Nam, "Power-sharing method of multiple distributed generators considering control modes and configurations of a microgrid," IEEE Trans. on Power Delivery, vol. 25, no. 3, pp. 2007-2016, 2010.

[12] H. Karimi, H. Nikkhajoei and R. Iravani, "Control of an electronicallycoupled distributed resource unit subsequent to an islanding event," IEEE Trans. on Power Delivery, vol. 23, no. 1, pp. 493-501, 2008.

[13] Xiaoxiao Yu, A. M. Khambadkone, Huanhuan Wang, and S. Terence, "Control of parallel-connected power converters for low-voltage microgrid-part I: A hybrid control architecture," IEEE Trans. on Power Electronics, vol. 25, no. 12, pp. 2962-2970, 2010.

[14] M. Hamzeh, H. Karimi and H. Mokhtari, "A new control strategy for a multi-bus mv microgrid under unbalanced conditions," IEEE Trans. on Power System, vol. 27, no. 4, pp. 2225-2232, Nov. 2012.

[15] F. Shahnia, R. Majumder, A. Ghosh, G. Ledwich and F. Zare, "Operation and control of a hybrid microgrid containing unbalanced and nonlinear loads," Electric Power System Research, vol. 80, no. 8, pp. 954 965, Aug. 2010.

[16] R.P.S. Chandrasena, F. Shahnia, S. Rajakaruna and A. Ghosh, "Control, operation and power sharing among parallel converter-interfaced DERs in a microgrid in the presence of unbalanced and harmonic loads," $23^{\text {rd }}$ Australasian University Power Engineering Conference (AUPEC), pp. $1-6,2013$.

[17] R. Majumder, F. Shahnia, A. Ghosh, G. Ledwich, M. Wishart and F. Zare, "Operation and control of a microgrid containing inertial and noninertial micro sources," IEEE Region 10 Conference (TENCON), pp. 16, 2009.

[18] A. Tewari, Modern Control Design with Matlab and Simulink, Wiley, 2002. 